\title{
MULTI-TEMPORAL CROP SURFACE MODELS COMBINED WITH THE RGB VEGETATION INDEX FROM UAV-BASED IMAGES FOR FORAGE MONITORING IN GRASSLAND
}

\author{
M. Possoch ${ }^{\text {a }}$, S. Bieker ${ }^{\text {b }}$, D. Hoffmeister ${ }^{\text {c }}$, A. Bolten ${ }^{\text {a }}$, J. Schellberg ${ }^{\text {d,e }}$, G. Bareth $^{\text {a }}$ \\ ${ }^{a}$ Institute of Geography, GIS \& RS Group, University of Cologne, Albertus-Magnus-Platz, 50923 Cologne, Germany - \\ mpossoch@smail.uni-koeln.de, (andreas.bolten, g.bareth)@uni-koeln.de \\ ${ }^{\mathrm{b}}$ Institute of Crop Science and Resource Conservation, Crop Science Group, University of Bonn, Katzenburgweg 5, 53115 Bonn, \\ Germany - (s7sobiek@uni-bonn.de) \\ ${ }^{c}$ GIScience Research Group, Institute of Geography, Heidelberg University, Berliner Straße 48, Heidelberg 69120, \\ Germany - hoffmeister@uni-heidelberg.de \\ ${ }^{\mathrm{d}}$ Institute of Crop Science and Resource Conservation, Agro- and Production Ecology, University of Bonn, Melbweg 42, 53127 \\ Bonn, Germany, j.schellberg@uni-bonn.de \\ ${ }^{\mathrm{e}}$ Center for Remote Sensing of Land Surfaces, University of Bonn, Walter-Flex-Str. 3, D-53113 Bonn, Germany
}

Commission I, ICWG I/Vb

KEY WORDS: Precision Agriculture, Grassland, UAV, Crop Surface Model, Structure from Motion, RGBVI

\begin{abstract}
:
Remote sensing of crop biomass is important in regard to precision agriculture, which aims to improve nutrient use efficiency and to develop better stress and disease management. In this study, multi-temporal crop surface models (CSMs) were generated from UAVbased dense imaging in order to derive plant height distribution and to determine forage mass. The low-cost UAV-based RGB imaging was carried out in a grassland experiment at the University of Bonn, Germany, in summer 2015. The test site comprised three consecutive growths including six different nitrogen fertilizer levels and three replicates, in sum 324 plots with a size of $1.5 \times 1.5 \mathrm{~m}$. Each growth consisted of six harvesting dates. RGB-images and biomass samples were taken at twelve dates nearly biweekly within two growths between June and September 2015. Images were taken with a DJI Phantom 2 in combination of a 2D Zenmuse gimbal and a GoPro Hero 3 (black edition). Overlapping images were captured in 13 to $16 \mathrm{~m}$ and overview images in approximately $60 \mathrm{~m}$ height at 2 frames per second. The RGB vegetation index (RGBVI) was calculated as the normalized difference of the squared green reflectance and the product of blue and red reflectance from the non-calibrated images. The post processing was done with Agisoft PhotoScan Professional (SfM-based) and Esri ArcGIS. 14 ground control points (GCPs) were located in the field, distinguished by $30 \mathrm{~cm} \times 30 \mathrm{~cm}$ markers and measured with a RTK-GPS (HiPer Pro Topcon) with $0.01 \mathrm{~m}$ horizontal and vertical precision. The errors of the spatial resolution in $\mathrm{x}-, \mathrm{y}-, \mathrm{z}-$ direction were in a scale of 3-4 cm. From each survey, also one distortion corrected image was georeferenced by the same GCPs and used for the RGBVI calculation. The results have been used to analyse and evaluate the relationship between estimated plant height derived with this low-cost UAV-system and forage mass. Results indicate that the plant height seems to be a suitable indicator for forage mass. There is a robust correlation of crop height related with dry matter $\left(\mathrm{R}^{2}=0.6\right)$. The RGBVI seems not to be a suitable indicator for forage mass in grassland, although the results provided a medium correlation by combining plant height and RGBVI to dry matter $\left(\mathrm{R}^{2}=0.5\right)$.
\end{abstract}

\section{INTRODUCTION}

Remote sensing increasingly contributes to precision agriculture activities for monitoring agricultural crops and optimizing crop management, which aims to lead to a maximisation of yield and a minimisation of ecological damage (Charles et al., 2010). Compared to destructive sampling, remote sensing methods are non-destructive, are applicable in non-accessible areas, and provide a time-effective and low-cost solution. Therefore, it is possible to monitor the growth and vitality of plants, to estimate predictions, and to model growing parameters like yield, biomass, and nitrogen content (Pinter et al., 2003). Laboratory methods provide more precise results, but high costs and efforts are the consequences (Geipel et. al., 2014).

Surprisingly, low-cost remote sensing concepts of precision agriculture are barely applied in grassland systems. Most of the present research and technical development in precision agriculture concentrate on crops rather than on grassland (Schellberg et al., 2008).

However, the monitoring of grassland ecosystems is an important task for providing spatial information on forage mass and nutrition quality. For the interpretation of spectral response of grassland, which have often a high level of heterogeneity and spatial variability, data acquisition is of key importance (Schellberg et al., 2008, Bareth et al., 2015). Accurately quantifying forage mass is essential for an effective pasture management including the monitoring of forage vitality, the improvement of nutrient use efficiency, and the prediction of yields. Whereas in the past, field data as forage vitality and nutrition quality had to be taken invasively and in great extent to determine forage mass (Reddersen et al., 2014). Nowadays grassland parameters can easily be explored with modern sensor techniques, whilst field sampling and laboratory analysis can serve for calibration of the sensors and its generated data. Besides physical measurement methods providing ground truth data by using a Rising Plate Meter (RPM) or Pasture Meter, spectral measurement methods were established (Sharrow, 1984). Spectral sensors, which are installed on quads (Lawrence et al., 2007), ground based platforms (Hoffmeister et al., 2013; Tilly et al., 2014) or UAVs (Bendig et al., 2013; Bareth et al., 2015), can deliver e.g. information of plant height, which is proofed to serve as a suitable biomass estimator for crops (Bendig et al., 2015; Tilly et al., 2014). Commonly, biomass is 
still determined in grasslands by destructive sampling methods or with manual RPM measurements (Reddersen et al., 2014).

This research investigates the potential of low-cost UAVsystems for RGB image acquisition to spatially determine plant height and forage mass in managed grasslands. Therefore, two working packages are investigated: (i) the transfer of the concept of Crop Surface Models (CSMs) to derive plant height as an estimator for forage mass and (ii) the application of the vegetation index for the visible domain developed by Bendig et al. (2015), the RGBVI, which can be combined with plant height for the Grassland Index (GrassI) introduced by Bareth et al. (2015). For evaluation, extensive ground truth data were collected at a grassland field experiment of Campus KleinAltendorf (University Bonn).

\section{MATERIAL AND METHODS}

\subsection{Test Site}

The test site encompasses a field experiment with a nitrogen fertilizer gradient on permanent grassland in Ersdorf, near Meckenheim, being part of the Campus Klein-Altendorf, Rheinische Friedrich Wilhelms University, Bonn (N 50³4'56.4”, E 6 59'21.1"). The test site is located between 323 and $320 \mathrm{~m}$ above sea level (a.s.l.) having a south-east exposition. The experiment is organised in 324 plots, each with a size of $1.5 \times 1.5 \mathrm{~m}$, subdivided in a randomised order of six nitrogen fertilizer levels (N1-6), consisting of 18 plots and three replicates in each case (Fig. 1). The experiment was set up for three growths within one year, two growths are analysed in this paper (growth A2 \& A3). On each field campaign the whole study area was overflown by an UAV for RGB imaging and 18 sampling plots (three of each N-level) were measured by a spectrometer and were afterwards harvested for biomass analysis. Flight surveys and biomass sampling were carried out frequently, nearly biweekly from June to September (twelve dates), whereas on sample date 8 ( $26^{\text {th }}$ August) only biomass sampling without a flight survey was carried out. For image referencing, 14 ground control points (GCPs) were welldistributed across the field and were precisely measured with a RTK-GPS (providing $0.01 \mathrm{~m}$ horizontal and vertical precision.

\subsection{Experimental Setup}

All plots were fertilized with calcium ammonium nitrate $(\mathrm{CaN}$,
$27 \% \mathrm{~N}$ ) at the beginning of each growth (beginning of June and midmonth of August) and separated by $20 \mathrm{~cm}$ width border strips treated with herbicides. Six fertilizer levels were established from $0-500 \mathrm{~kg} \mathrm{~N} \mathrm{ha}^{-1}$ in steps of $100 \mathrm{~kg} \mathrm{~N} \mathrm{ha}^{-1}$. On each fertilization-date one quarter of this amount was given, as the experiment is treated in a three-cut-regime. For analysis of the nitrogen content, plots were harvested and analysed on six dates during each growth with a determined sub-plot area (Fig. 1). For each growth each plot was distinguished with a unique sample code from A1T1N1W1 to A3T6N6W3 to have an accurate encoding and to avoid an accidental harvesting of a wrong plot (s. Fig. 1).

Weather conditions in 2015 were almost average with a mean annual temperature of approx. $10.7^{\circ} \mathrm{C}$ and a precipitation sum of approx. $686 \mathrm{~mm}$, whereas Spring and July were too dry towards a wet August and September (data of weather station on Campus Klein-Altendorf, approx. $190 \mathrm{~m}$ a.s.l., 3-4 km distance to experiment).

\subsection{Biomass Sampling}

Destructive samples of above ground biomass from the 18 plots $(3 \times 6 \mathrm{~N}$-levels), which were clearly defined and encoded for each date, were taken at 12 dates nearly biweekly (sample dates in Tab. 1). The sampling dates were done within a maximum of two days after the flight surveys and the field spectroradiometer measurements. The harvesting was conducted with the same sickle bar mower with a cutting width of $1.2 \mathrm{~m}$ along the complete plot length. The plot border strips caused slightly different plot sizes between 1.25 and $1.40 \mathrm{~m}$ length, so the plot lengths had to be rechecked and the results of the yield extrapolation were corrected. For the fresh biomass, the samples were weighed and sub-sampled in approx. $300 \mathrm{~g}$ amounts. In sum, 18 samples per date were processed and afterwards dried at $60{ }^{\circ} \mathrm{C}$ for at least $168 \mathrm{~h}$ (one week) in a dry oven. And then dry biomass was weighed again. The weights were extrapolated to tons per hectare $\left[\mathrm{tha}^{-1}\right]$ for analysis.

\subsection{Applied Methods of Remote Sensing}

In this study, a low-cost UAV equipped with a Gimbal and a customary, non-calibrated GoPro RGB-camera was used. The multi-temporal RGB data were used to compute vegetation indices, which were evaluated against spectral field masurements and against destructively sampled plant

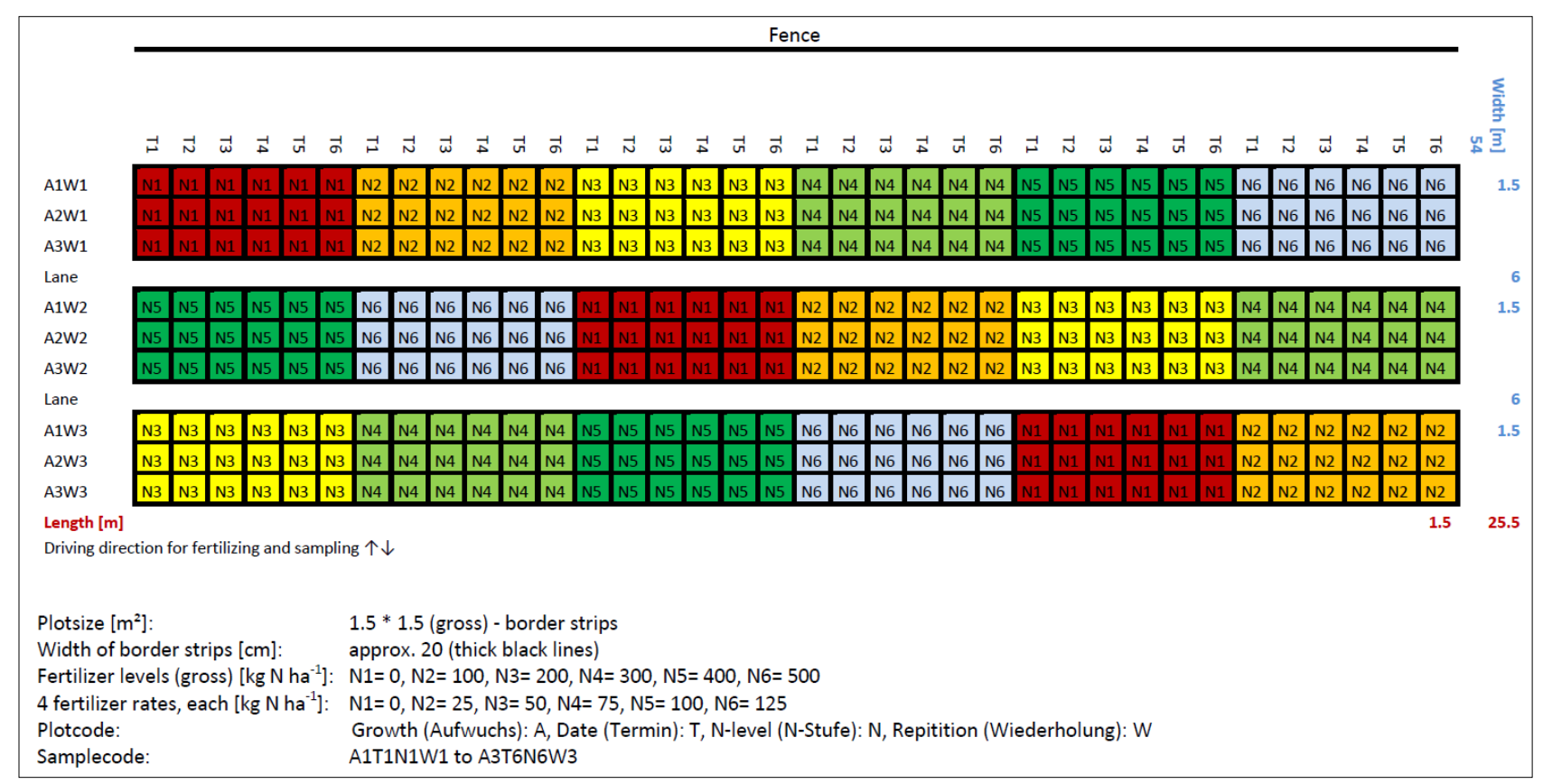


parameters. Non-destructive measurements were acquired using an UAV and a correlation analysis was used to quantify the strength of the relationship between plant height and biomass. There are several established methods for biomass estimation: Calculation of vegetation indices (VIs), especially in the near infrared region (NIR) are applied (Bannari et al., 1995). Moreover, in recent years biomass was directly modelled by using plant height information. The suitability of this method is well-researched for several crops like wheat, oat, barley, sugar beet and paddy rice (Tilly et al., 2014), but not sufficiently researched in grassland (Bareth et al., 2015). The advantage of this method is the possibility of a high spatial and temporal resolution, which is delivered by multi-temporal crop surface models (Hoffmeister et al., 2010; Bendig et al., 2013; Bendig et al., 2015). In Hoffmeister et al. (2013) and Tilly et al. (2014) a ground-based data collection platform with a terrestrial laser scanner was used. A more time- and also cost-effective and flexible data collection method is an airborne platform using an UAV and a RGB camera (Bendig et al., 2013). Because of the possibility of low flight heights, the high resolution of current RGB-cameras and more efficient and detailed working software, UAV-acquired images can achieve a cm-resolution and furthermore the RGBVI can be calculated from the RGB images (Bendig et al., 2015). As mentioned before, UAV imaging approaches to monitor forage mass in grassland are rare and not well investigated. A combination of estimated plant height and RGBVI from the same dataset of UAV-based noncalibrated RGB imagery suggests an improvement of biomass estimation in the field of crop monitoring by implementing suitable regression models (Bendig et al., 2015).

\subsubsection{UAV and Photogrammetry:}

The UAV used in this study was a DJI Phantom 2 with a 2D Zenmuse gimbal (SZ DJI Technology Co., Shenzhen, China) and a GoPro Hero 3 black edition (Go Pro Inc., San Mateo, California, USA) without a GPS-controlled flight route. The gimbal compensates the UAV movement (pitch and roll) during the flight and guarantees close to nadir image collection (Bendig et al., 2014). So the RGB images were acquired using a GoPro Hero 3 black edition in a nadir position (vertical) with a standard resolution of $3,000 \times 4,000(12$ million $)$ pixels. The camera lens has a fish eye effect, which leads to a distortion towards the outer areas of the lens. In addition to the main research question is the question whether the distortion influences or interrupts the calculation of the crop surface models. The total price of this low-cost UAV-system was approx. 1,300 Euro in 2014. The images were captured in an automatic mode at 2 frames per second [fps]. In each flight survey overlapping images and overview images were recorded. Overlapping images were captured in 13-16 meters height and overview images at approximately $60 \mathrm{~m}$ above ground level (AGL) on each date around $11.30 \mathrm{am}$ local time. On each flight date between 800 and 1,200 photos of the experiment were taken. Multi-temporal crop surface models (CSMs) were generated from UAV-based dense imaging in order to derive plant height distribution and to determine forage mass. On $9^{\text {th }}$ June 2015 the bare ground model was recorded (basis model). Determining the plant height by taking the difference between a crop surface model and an existing digital terrain model (DTM) by an initial flight survey has the advantage that the reference surface is always the same and the plant height measurement is accurate and reliable throughout the entire growing season (Grenzdoerffer, 2014). Within the second growth (A2) six flight dates $\left(26^{\text {th }}\right.$ June $-31^{\text {st }}$ July 2015$)$ and within third growth (A3) five flight dates $\left(20^{\text {th }}\right.$ August $-24^{\text {th }}$ September 2015) were carried out simultaneously to the sample dates. The images were recorded during homogeneous and stable solar illumination conditions, except one date: On sample date 3 within second growth $\left(8^{\text {th }}\right.$ July 2015 , A2) changing conditions between sunny and cloudy occurred, nevertheless images were used.

Approximately 400 photos were used of each date to compute the multi-temporal CSMs with the software Agisoft PhotoScan Professional 1.1.6 (Agisoft LLC, St. Petersburg, Russia). In the background of this specific software multi-view stereopsis-algorithms (MVS) were recalculating the matching points of the images (Verhoeven, 2011). The software is using a so-called mosaicing blending mode to produce additional orthophotos using a pixel value of the most appropriate photo (Agisoft LLC, 2014). In a next step the GCP-positions were imported to get a precise orthorectified image (Lucieer et al., 2014). For each of the 14 GCP-positions at least 5 coordination references were manually placed (s. Fig. 2). The command 'optimize cameras' were used for achieving a higher accuracy. Matching features in the images resulting from a bundle adjustment in a composite photo (Sona et al., 2014). In a last step, a digital surface model in *TIF image format was exported, which contains the crop surface model (CSM) information at a cm-resolution (Bendig et al., 2015).

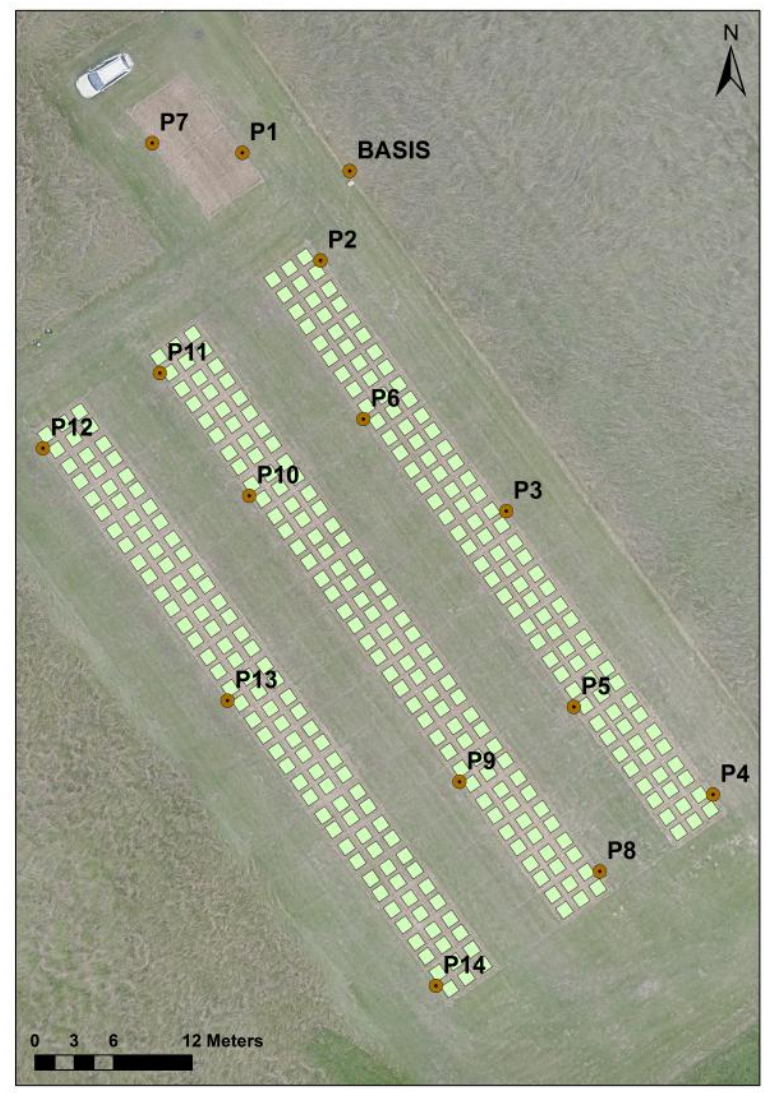

Figure 2. Composite photo of basis date ( $9^{\text {th }}$ June 2015) with polygons and GCPs

The analysis of plant height and the RGBVI was done with Esri ArcGIS ArcMap 10.3 (Esri, Redlands, California, USA). The composite photos of each date were imported into the software and transferred to a raster file (Bendig et al., 2013). Polygons (s. Fig. 2) were manually digitized for each plot with an inside buffer of $0.2 \mathrm{~m}$ to the outlines and saved as a shapefile to serve as mask zones for zonal statistics (Bendig et al., 2013). The buffering was made because of existing border effects between differently fertilized plots (Bareth et al., 2015). The GCPs were identified in the images and used for georeferencing. A basic 
Table 1. Overview of number and features of photos of all sample dates

\begin{tabular}{|c|c|c|c|c|c|c|}
\hline Sample Date & Date & Photos & Error ReferenceTotal [m] & Error ReferenceTotal [pix] & Ground Resolution $[\mathrm{m} / \mathrm{pix}]$ & Resolution DEM [m/pix] \\
\hline Basis & 09.06 .2015 & 383 & 0.033 & 0.41 & 0.01 & 0.02 \\
\hline 1 & 26.06 .2015 & 436 & 0.029 & 0.24 & 0.008 & 0.017 \\
\hline 2 & 01.07 .2015 & 436 & 0.035 & 0.27 & 0.007 & 0.015 \\
\hline 3 & 08.07 .2015 & 436 & 0.037 & 0.27 & 0.008 & 0.017 \\
\hline 4 & 16.07 .2015 & 430 & 0.036 & 0.2 & 0.007 & 0.014 \\
\hline 5 & 23.07 .2015 & 349 & 0.035 & 0.14 & 0.007 & 0.014 \\
\hline 6 & 31.07 .2015 & 434 & 0.034 & 0.16 & 0.007 & 0.015 \\
\hline 7 & 20.08 .2015 & 431 & 0.035 & 0.17 & 0.007 & 0.015 \\
\hline 8 & only biomass sampling & & & & & \\
\hline 9 & 02.09 .2015 & 441 & 0.033 & 0.16 & 0.008 & 0.017 \\
\hline 10 & 09.09 .2015 & 408 & 0.03 & 0.11 & 0.008 & 0.016 \\
\hline 11 & 18.09 .2015 & 432 & 0.04 & 0.17 & 0.006 & 0.012 \\
\hline 12 & 24.09 .2015 & 423 & 0.032 & 0.1 & 0.007 & 0.013 \\
\hline Mean & & 419 & 0.034 & 0.2 & 0.008 & 0.015 \\
\hline
\end{tabular}

ground model was recorded on the ninth of June 2015, directly after an entire cut of the experiment field. Plant height was computed with ArcGIS by subtracting the basis DTM from the CSM for each date (Bendig et al., 2014). To derive mean plant height for each plot and for each date zonal statistics was used. In Table 1 an overview of number and features of composite photos of the basis date and twelve sample dates is shown, whereby on sample date eight only biomass was collected. Within each flight survey it was paid attention to get a sufficient number of photos for the aligning process. For the resulting composite photo for each sample date, around 400 photos were chosen to acquire an optimal coverage of the experiment field and the GCPs. The error reference (mean error of 14 GCPs for each date from Agisoft Photoscan report file) of these photos fluctuated in a range between three and four centimetres. The error reference in pixels amounts in mean of 0.2 pixels. The ground resolution amounts in mean 0.008 meter per pixel and the resolution of DEM 0.015 meter per pixel. In relation to the size of the experiment field these errors are representing a suitable performance of this low-cost system and this appropriate approach.

The RGBVI was not calculated from the orthophotos but from a separately acquired overview photo covering the complete field experiment in one image. This approach was chosen because of a higher resolution towards the composite photos reducing the risk of changing irradiation conditions. Due to the usage of a GoPro camera, it was important to correct the fisheye distortion for accuracy reasons. For this correction the software PTLens (T. Niemann, Portland, Oregon, USA) was used, which has an ability to correct lens pincushion, barrel distortion, vignetting, chromatic aberration and perspective. The overview photos were georeferenced with the GCPs. so that a superimposing on the other photos is automatically realised. The RGBVI was calculated with the raster calculator in ArcGIS.

\subsubsection{Spectral Measurements:}

In addition to biomass sampling and flight surveys, spectral reflectance measurements were collected with the handheld spectrometer Ocean Optics QE 65000 (Ocean Optics Inc., Dunedin, Florida, USA). This device has a spectral range from $350 \mathrm{~nm}$ to $1,150 \mathrm{~nm}$ and an optical resolution of $0.81 \mathrm{~nm}$. At each date the measurements were carried out at about 11 am to ensure nearly the same solar irradiation conditions for wellcomparable results. As said before, constant solar conditions were a requirement for each measuring date. Unfortunately, it turned cloudy (Cirrus clouds) on sample date 9 ( $2^{\text {nd }}$ September $)$.

\subsubsection{Compressed Sward Height (CSH-PH):}

During the second growth, ground truth measurements were acquired for a supportive validation of plant height and biomass. Compressed sward height (CSH-PH) was measured by a mechanic rising plate meter (RPM). The rising plate meter is constructed of a $40 \mathrm{~cm}$ diameter wide aluminium disc, which has a hole in the centre where a meter stick is passing. Plant height is determined by lowering the disc along the meter stick until the canopy surface is in contact with the disc and can be read directly off the meter stick (Sharrow, 1984). Four measurements were taken in each determined plot within the buffer zone by placing the plate gently on the grass sward until the plate was supported. The forage is compressed only by the weight of the disc and that is the value, which was read off as the height of the plate's top above the ground. The measurements has started on sample date $5\left(23^{\text {rd }} \mathrm{July}\right)$, at the end of the second growth. Per plot mean values from four measurements were calculated to represent the plant height for a plot as a function of compressed sward height.

\subsection{RGBVI Analysis}

The RGBVI was introduced by Bendig et al. (2015) as the normalised difference of the squared green reflectance and the product of bluexred reflectance with the function of capturing reflectance differences between chlorophyll a-absorption and chlorophyll b-absorption:

$$
R G B V I=\frac{G^{2}-(R * B)}{G^{2}+(R * B)}
$$

The RGBVI was calculated on the basis of the RGB imagery and for the field spectra with simulated RGB bands of WorldView 2 (red: $630-690 \mathrm{~nm}$, green: $510-580 \mathrm{~nm}$, blue: 450 $510 \mathrm{~nm}$ ). Overview images were postprocessed in Esri ArcGIS 10.3, whilst three single band files (red, green, blue) were created and set against each other by using the command 'raster calculator'. And analogue to the CSM-PH, RGBVI images were created for each date and a mean for each plot was calculated by the command 'zonal statistics as table'. Additionally, the RGBVI was calculated using the data from hyperspectral measurements to provide a calibrated RGBVI for statistical analysis.

The potential of this low-cost approach is analysed by using different validation methods (ground truth data, calibrated hyperspectral measurements) and correlation constellations. Further calculations and statistical analyses were executed in Microsoft Excel 2013 (Microsoft Corp., Redmond, Washington, USA).

\section{RESULTS}

Following figures are showing the complete data set of each parameter. Linear models are underlain, because of relatively low plant heights. Forage mass is indicated by dry matter in tons per hectare. Outliers were out of range of a $95 \%$ confidence interval, in sum a maximum of two outliers were detected: On sample date 1 with $0.5 \mathrm{t} \mathrm{ha}^{-1}$ and on sample date 12 with $4.5 \mathrm{t} \mathrm{ha}^{-1}$ dry matter. 


\section{Crop Surface Models}

By setting the plant height from Crop Surface Model (CSM-PH) in meters in relation to dry matter (DM) in tons per hectare (Fig. 3 ) a good correlation is achieved. For the analysis, presented in Fig. 3, no outliers but all other values from growth $2 \& 3$ (A2 \& A3) are used and result in a $\mathrm{R}^{2}$ of 0.64 with a number of 196. In this actual case 10 centimetres plant height are equal to 1.5 tons per hectare dry matter. The maximum of measured and reliable results are $3.2 \mathrm{t} / \mathrm{ha}$ dry matter and 26 centimetres plant height. The spread is increasing with higher plant heights and dry matter values. The formula of the regression line represents a rough affiliation between particular plant heights with dry matter quantities for this special area and case.

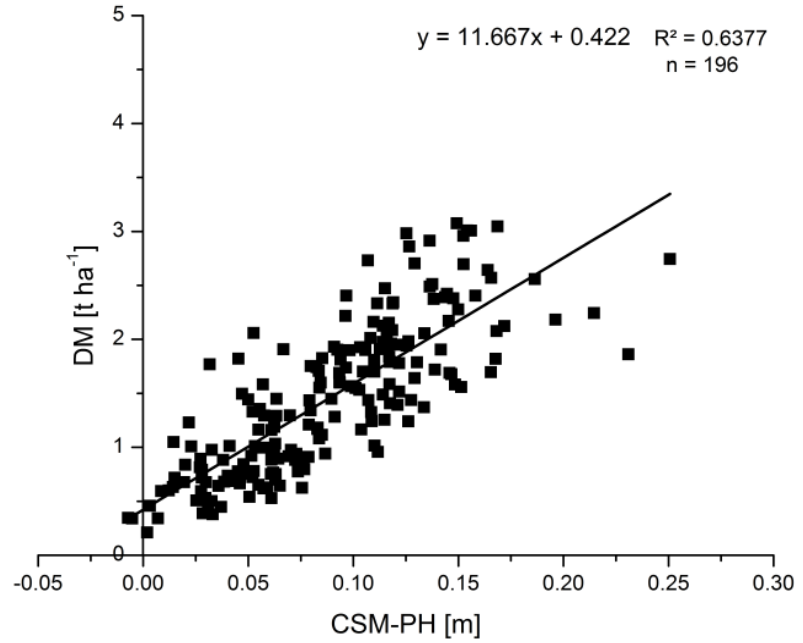

Figure 3. Plant height from Crop Surface Model (CSM-PH) versus dry matter (DM), Growth $2 \& 3$ (A2 \& A3)

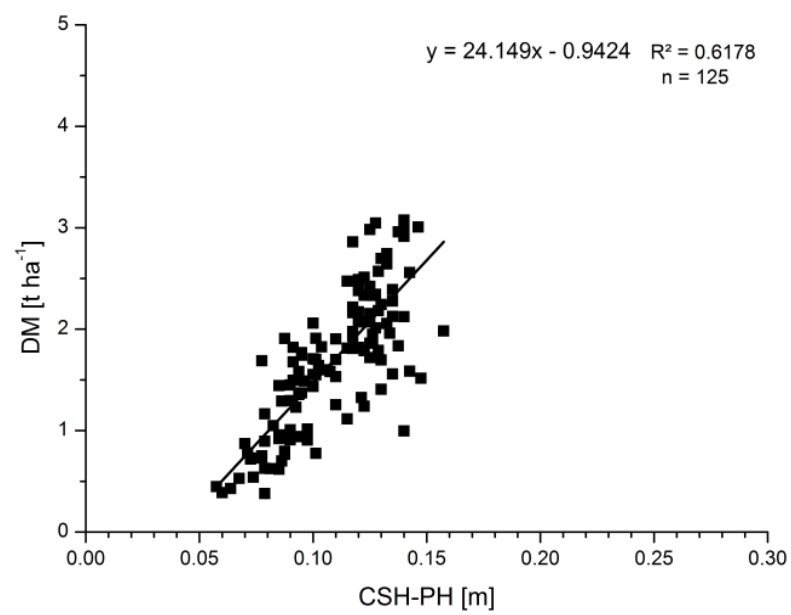

Figure 4. Plant height from compressed sward height (CSH$\mathrm{PH})$ versus dry matter (DM), G 2 \& 3

In comparison to CSM-PH the plant height from compressed sward height (CSH-PH, rising plate meter measurements) in meters in relation to dry matter again without relevant outliers of all recorded values from Growth 2 \& 3 (A2 \& A3) are figured in Figure $4 . R^{2}$ amounts here 0.62 with a number of 125 . By contrast to plant height of CSM, $\mathrm{R}^{2}$ is a little bit lower and obviously measurement problems in low plant heights are appearing, because of stems, which are preventing measurements in relation to the direct ground surface. Maximum values of 16 centimetres compressed sward height were measured.

To have a direct comparison between the results of the two plant height estimation methods, CSM-PH in meters in relation to $\mathrm{CSH}-\mathrm{PH}$ in meters of all comparable values of Growth $2 \& 3$ (A2 \& A3) are depicted in Figure 5.

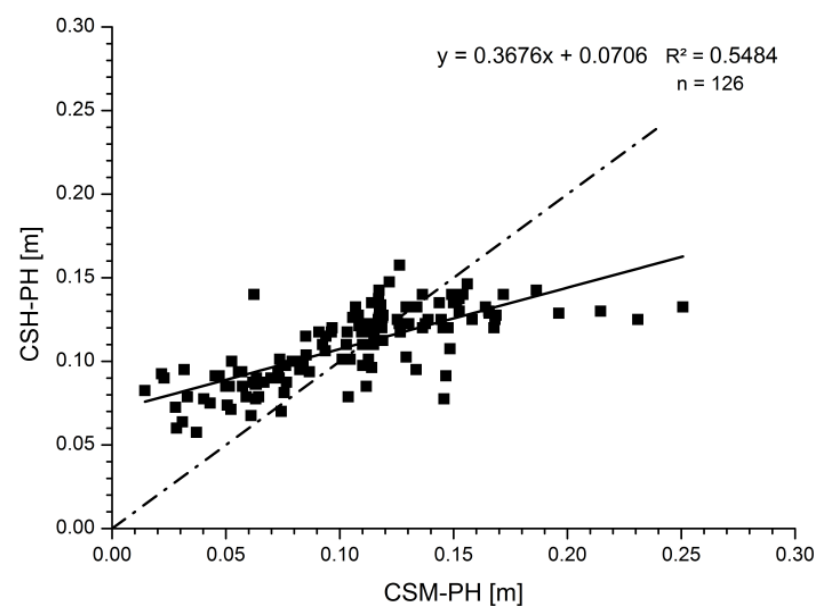

Figure 5. Plant height from Crop Surface Model (CSM-PH) versus plant height from compressed sward height (CSH-PH), G $2 \& 3$

The correlation is specified with a $\mathrm{R}^{2}$ of 0.55 with a number of 126. In comparison to 1:1 line, regression line has a lower slope and the matching point is located in region of approx. 12 centimetres. It seems that there is an underestimation of plant height in low levels and overestimation of plant height in higher levels of CSM in relation to CSH. Taking into account that the plant height is compressed by the rising plate meter, the plant height derived from CSM is probably closer to reality in height levels above $0.15 \mathrm{~m}$. But the higher grass is growing, the more the effect of lodging is influencing the plant height. And there isn't a trivial relationship of plant height to density and homogeneity of the grassland area.

\section{RGBVI}

In addition to the plant height correlation with dry matter, the RGBVI correlation to dry matter was investigated.

In Figure 6a the RGBVIs from overview photos related to dry matter $(\mathrm{DM})$ in tons per hectare are displayed and the RGBVIs from hyperspectral measurements related to dry matter in tons per hectare with all values of growth $2 \& 3$ (A2 \& A3) is depicted in Figure 6b. 


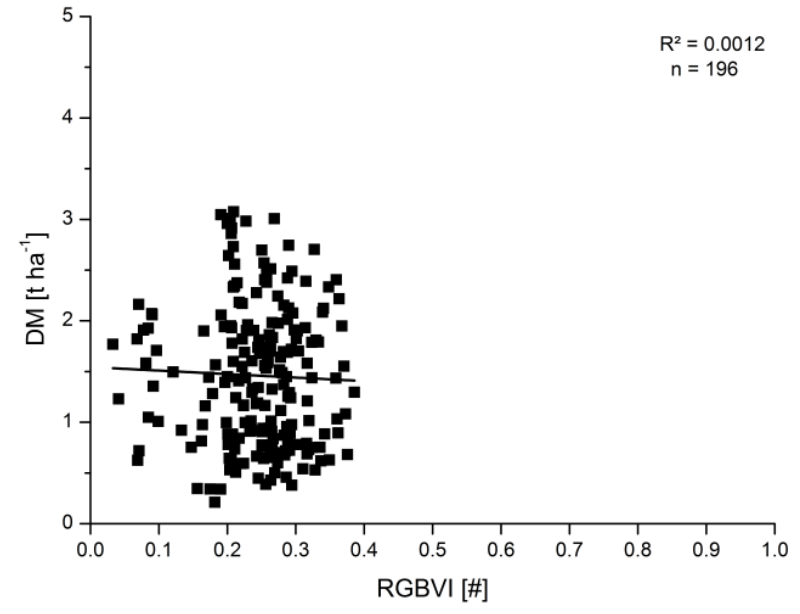

Figure 6a. Red Green Blue Vegetation Index (RGBVI) from overview photos versus dry matter (DM) in tons per hectare, $\mathrm{G}$ $2 \& 3$

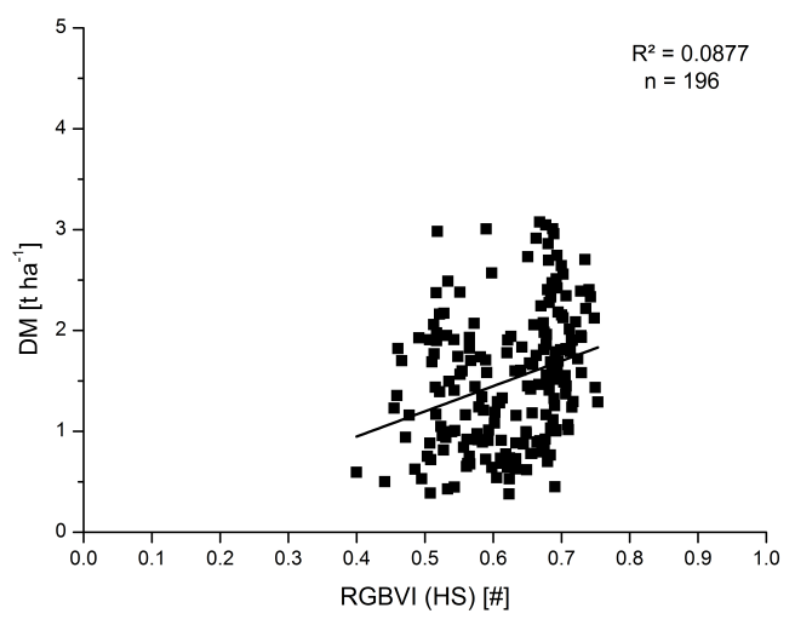

Figure 6b. RGBVI from hyperspectral measurements versus dry matter (DM), G $2 \& 3$

It is noticeable that the RGBVIs from the photos are mostly in a range between 0.1 and 0.4 , whereas the RGBVIs from hyperspectral measurements are mostly in a range from 0.4 to 0.8 . Based on the $\operatorname{CoD}\left(\mathrm{R}^{2}=0.001 ; \mathrm{R}^{2}=0.09\right)$ and the shape of the point clouds, no reliable correlation between the variables was found.

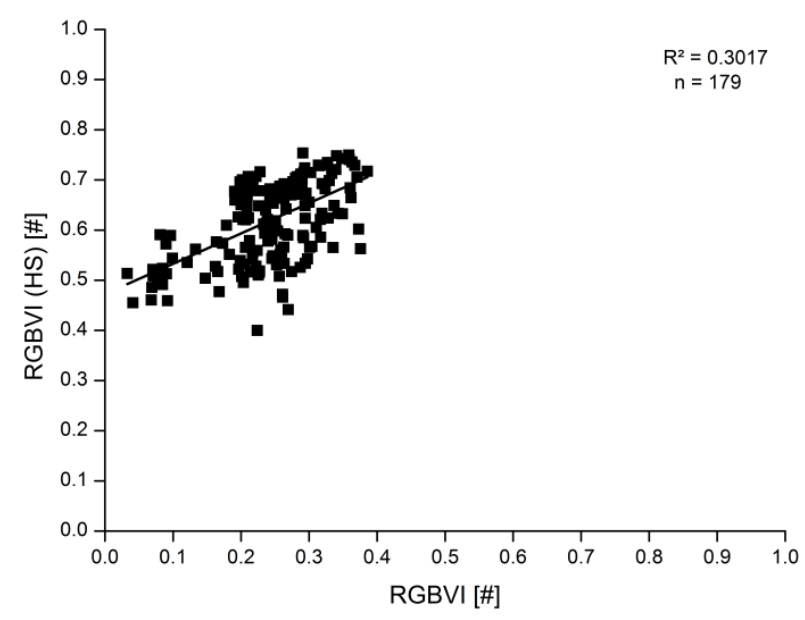

A direct comparison of the different measured RGBVIs is plotted in Figure 7, in which the RGBVIs from overview photos are compared to the RGBVIs from hyperspectral measurements with all comparable values of Growth $2 \& 3$ (A2 \& A3).

With a number of 179 the $\mathrm{R}^{2}$ is 0.3 and an acceptable slope of the regression line, this conveys very clearly that the value range of the differently computed RGBVIs are not related.

\section{GrassI}

A combination of plant height and RGBVI is represented by the so-called Grassland Index (GrassI) introduced by Bareth et al. (2015).

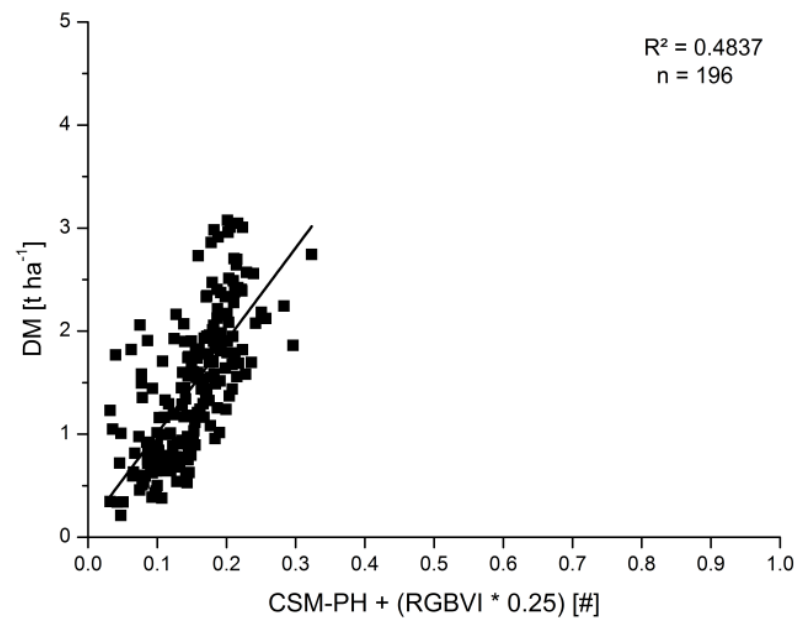

Figure 8a. GrassI from photos (CSM-PH aggregated with $25 \%$ of each RGBVI value) versus dry matter (DM), G $2 \& 3$

In Figure 8a, GrassI-Indices from photos are represented by CSM-PH aggregated with $25 \%$ of each RGBVI value to equalize the range values of CSM-PH and RGBVI in relation to dry matter (DM) in tons per hectare.

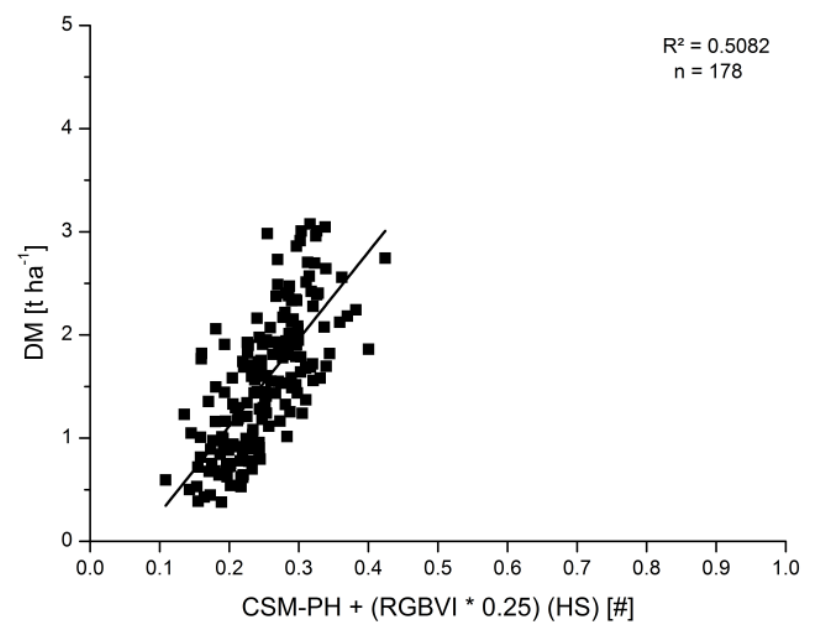

Figure 8b. GrassI-Index from hyperspectral measurements (CSM-PH aggregated with $25 \%$ of each RGBVI value) versus dry matter (DM), G 2 \& 3

In Figure $8 b$ in an analogue way the GrassI-Indices from hyperspectral measurements in relation to dry matter in tons per hectare with all comparable values of Growth $2 \& 3$ (A2 \& A3). By comparing the $\mathrm{CoD}$ of the combination of plant height and RGBVIs from photo data $\left(\mathrm{R}^{2}=0.48, \mathrm{n}=196\right)$ and of the combination of plant height and RGBVIs from hyperspectral data $\left(R^{2}=0.51, n=178\right)$, it is recognisable that there are only

Figure 7. RGBVI from overview photos versus Red Green Blue Vegetation Index from hyperspectral measurements, G 2 \& 3 
slight differences. However, the combination with hyperspectral data achieved higher correlations.

\section{DISCUSSION}

The primary aim of this study was to evaluate the potential of low-cost UAV-based RGB imaging and its products the CSMderived plant height $(\mathrm{PH})$ and RGBVI for forage mass estimation of grasslands. Intensive destructive and nondestructive groundtruth were collected for evaluation analysis. In contrast to other studies, the performance of CSM-derived plant height and the one of uncalibrated and calibrated RGBVI for forage mass estimation is investigated. Finally, we conducted an evaluation of the GrassI, which combines plant height and RGBVI. To get useable images and comparable results, solar irradiation conditions, flight height and the flight route (with the related overlap area and the offset) were quite similar within each flight survey and across all flight surveys. In general, the automation process in aerial triangulation and the geometric quality of the final product becomes more reliable with a large amount of high-quality images and a large overlap area, because of higher correlations between adjacent image frames (Rokhmana, 2015). The process to get the information of plant height from RGB-images by using Agisoft photoscan software worked widely unproblematic. The results of mean calculation of plant height (CSM-PH) for each plot looked visually realistic and the validation by comparing the data with ground truth measurements (CSH-PH) confirmed the visual impression. But there occurred an underestimation at low plant heights and overestimation at higher plant heights of CSM in relation to $\mathrm{CSH}$, which can be partly explained by the approach of compressing the canopyto derive $\mathrm{CSH}$. Evidently this method has weaknesses especially in low plant heights, because related errors are comparatively high. As a rough guide 10 centimetres plant height are equal to 1 ton per hectare dry matter, in this actual case $10 \mathrm{~cm}$ are equal to $1.5 \mathrm{t} \mathrm{ha}^{-1}$ (DESTATIS, 2015). The results of the statistical analysis indicate that the plant height seems to be a suitable indicator of forage mass by showing a robust correlation of crop height (CSM-PH) related to dry matter $\left(\mathrm{R}^{2}=0.64\right)$, which is even slightly better than the correlation between ground truth measurements (CSH-PH) and dry matter $\left(\mathrm{R}^{2}=0.62\right)$. An explanation for the good performance of CSM-PH could be that it is also a kind of compressed plant height (Bareth et al., 2016, in print). However, the good results for CSM-PH states the findings by Bendig et al. (2014) and Tilly et al. (2014) but in the two crop studies the performance was better. The difference can be explained by the range of investigated plant height and the error of the method. While in crops like barley or wheat, the investigated plant height range is approx. $1 \mathrm{~m}$, in our grassland study it is only approx. $0.2 \mathrm{~m}$. Considering the SD of the CSM approach, the method performed surprisingly better than expected. However, further studies can examine correction factors to differentiate parameters in growing situations under specific conditions.

Since the GoPro lens has a fish eye effect causing a distortion of the overview photos, problems occurred within georeferencing and superimposing. According to Rokhmana (2015), the use of non-metric cameras is one of the most frequent errors of accuracy depending on the quality of the nonmetric lens. The specific usage of rectifying software like PTLens can solve these problems. Additionally, there is no radiometric correction between single images, which were taken under different irradiation conditions and even in close to nadir imaging, bidirectional reflectance distribution function (BRDF) effects are occurring (Grenzdoerffer \& Niemeyer, 2011; Bendig et al., 2015). That is the reason for using overview photos for the RGBVI calculation, which can partly eliminate the problem.
Nevertheless, RGBVI values can vary due to different irradiation conditions. Although it was highly considered that flight surveys were performed under similar irradiation conditions and overview photos were chosen with intra-uniform radiation ratios, problems of comparability are likely. But even the calibrated RGBVIs (from spectroradiometer) are not showing the desired correlation to biomass. So the potential of RGBVI, both from photos and hyperspectral measurements, to indicate forage mass could not be validated. The combination of plant height and RGBVI in relation to dry matter showed a medium correlation $\left(\mathrm{R}^{2}=0.5\right)$, but the practical applicability to estimate forage mass should be doubted.

By contrast the approach of obtaining plant height information from UAV-based RGB imagery and the estimation of forage mass and related results inclining to further research with validation and establishing this as a simple utilised method. This low-cost approach can be operated by an increasing number of user groups nowadays, can capturing greater areas and the spatial variability faster with a dense and complete spatial coverage than direct infield measurements (Bendig et al., 2015). The application potential is intensively rising by combining the advantages of UAV-features like simplicity of usage, time- and cost-effectiveness and the possibility to record great and hardly accessible areas with more precise and accurate non-calibrated RGB cameras and calibrated hyperspectral cameras (Aasen et al., 2015). These factors are opening up new possibilities in grassland and crop monitoring and help to improve the robustness and applicability of the correlation between plant height and biomass and a combination of diverse information sources in relation to biomass. Additionally, the higher the spatial resolution in future is, the higher the possibility will be to distinguish between different grassland types and spatial heterogeneity in fields and to fly in higher altitudes, which means even larger grassland areas can be covered (Bareth et al., 2015).

\section{CONCLUSIONS}

In this study the potential of low-cost UAV-based RGB image acquisition for spatially determining plant height and forage mass in a grassland experiment is examined. The UAV-derived plant height (CSM-PH) performs well and is evaluated against intensive ground truth measurements with a Rising Plate Meter which produces compress sward height (CSH-PH). Both, CSM$\mathrm{PH}$ and $\mathrm{CSH}-\mathrm{PH}$, are investigated as estimators for forage mass and both perform similar well (CSM-PH: $\mathrm{R}^{2}=0.64$; CSH-PH: $\mathrm{R}^{2}=0.62$ ). Therefore, we conclude that UAV-derived plant height from multi-temporal CSMs can serve as a good estimator for forage mass. Similar results are provided by Bareth et al. (2015) for a long-term grassland experiment. However, this method also has weaknesses for low plant heights, because related errors are comparatively high. Furthermore, the fish eye effect of the lens results in distorted photos, which caused problems for georeferencing and superimposing, but the usage of rectifying software solutions could eliminate these problems. From our results, the RGBVI, both from photos and hyperspectral measurements, has no potential to serve as an estimator for forage mass. Additionally, the combined analysis of plant height and RGBVI to estimate forage also performs weak in our study. However, a low-cost UAV-system with a non-calibrated camera can be used to estimate forage mass of grasslands with multi-temporal CSMs to determine plant height as a robust estimator for forage mass. 


\section{REFERENCES}

Agisoft LLC, 2014. Agisoft PhotoScan. URL http://www.agisoft.com (accessed 15.12.15)

Bareth, G., Bolten, A., Hollberg, J., Aasen, H., Burkart, A. \& Schellberg, J., 2015. Feasibility study of using non-calibrated UAV-based RGB imagery for grassland monitoring: Case study at the Rengen Long-term Grassland Experiment (RGE), Germany. In: DGPF Tagungsband 24/2015.

Bareth, G., Bendig, J., Tilly, N., Hoffmeister, D., Aasen, H. \& Bolten, A., 2016 (accepted). Comparison of UAV- and TLSderived Plant Height for Crop Monitoring: Using Polygon Grids for the Analysis of Crop Surface Models (CSMs). PFG.

Bendig, J., Bolten, A. \& Bareth, G., 2013. UAV-based Imaging for Multi-Temporal, very high Resolution Crop Surface Models to monitor Crop Growth Variability. PFG Photogrammetrie, Fernerkundung, Geoinformation 2013 (6), 551-562.

Bendig, J., Bolten, A., Bennertz, S., Broscheit, J., Eichfuss, S., \& Bareth, G., 2014. Estimating Biomass of Barley Using Crop Surface Models (CSMs) Derived from UAV-Based RGB Imaging. Remote Sensing 2014 (6), 10395-10412.

Bendig, J., Yu, K., Aasen, H., Bolten, A., Bennertz, S., Broscheit, J., Gnyp, M. L., \& Bareth, G., 2015. Combining UAV-based plant height from crop surface models, visible, and near infrared vegetation indices for biomass monitoring in barley. International Journal of Applied Earth Observation and Geoinformation 2015 (39), 79-87.

Charles, H., Godfray, J., Beddington, J. R., Crute, I. R., Haddad, L., Lawrence, D., Muir, J. F., Pretty, J., Robinson, S., Thomas, S. M. \& Toulmin, C., 2010. Food Security: The Challenge of Feeding 9 Billion People: A review. Science 2010 (327), 812818.

DESTATIS, 2015. Land use in Germany. https://www.destatis.de/DE/ZahlenFakten/Wirtschaftsbereiche/ LandForstwirtschaftFischerei/FeldfruechteGruenland/Methoden /AnleitungEBE.pdf?_blob=publicationFile (accessed 12.01.16)

Geipel, J., Link, J. \& Claupein, W., 2014. Combined Spectral and Spatial Modeling of Corn Yield Based on Aerial Images and Crop Surface Models Acquired with an Unmanned Aircraft System. Remote Sensing 2014 (11), 10335-10355.

Grenzdoerffer, G. J., 2014. Crop Height Determination with UAS Point Clouds. ISPRS Archives: ISPRS Technical Commission I, ICWG I/Vb, 135-140.

Hoffmeister, D., Bolten, A., Curdt, C., Waldhoff, G. \& Bareth, G., 2010. High resolution Crop Surface Models (CSM) and Crop Volume Models (CVM) on field level by terrestrial laserscanning. In: Proc. SPIE, Vol. 7840, 78400E: 6 p.

Hoffmeister, D., Waldhoff, G., Curdt, C., Tilly, N., Bendig, J. \& Bareth, G., 2013. Spatial variability detection of crop height in a single field by terrestrial laser scanning. In: Stafford, J.V. (Ed.), Precision Agriculture '13. Presented at the 9th European Conference on Precision Agriculture. Wageningen Academic Publishers, Lleida, Spain, 267-274.

Lawrence, H., Yule, I. \& Murray, R., 2007. Pasture Monitoring Technologies. Proceedings of the SIDE Conference 8, 126-131.
Lucieer, A., Jong, S. M. D. \& Turner, D., 2014. Mapping landslide displacements using Structure from Motion (SfM) and image correlation of multi-temporal UAV photography. Progress in Physical Geography 38 (1), 97-116.

Grenzdoerffer, G. J., Niemeyer, F., 2011. UAV based BRDFMeasurements of Agricultural Surfaces with PFIFFIKUS. International Archives of the Photogrammetry, Remote Sensing and Spatial Information Sciences, Vol. XXXVIII-1/C22 UAV-g 2011, Conference on Unmanned Aerial Vehicle in Geomatics, Zurich, Switzerland

Pinter, P. J. Jr., Hatfield, J. L., Schepers, J. S., Barnes, E. M., Moran, M. S., Daughtry, C. S. T. \& Upchurch, D. R., 2003. Remote Sensing for Crop Management. Photogrammetric Engineering \& Remote Sensing 69 (6), 647-664.

Reddersen, B., Fricke, T. \& Wachendorf, M., 2014. A multisensor approach for predicting biomass of grassland. Computers and Electronics in Agriculture 2014 (109), 247-260.

Rokhmana, C. A., 2015. The potential of UAV-based remote sensing for supporting precision agriculture. Procedia Environmental Sciences 2015 (24), 245-253.

Schellberg, J., Hill, M. J., Gerhards, R., Rothmundd, M. \& Braune, M., 2008. Precision agriculture on grassland: Applications, perspectives and constraints. European Journal of Agronomy 2008 (29), 59-71.

Sharrow, S. H., 1984. A Simple Disc Meter for Measurement of Pasture Height and Forage Bulk. Journal of Range Management 37 (1), 94-95.

Sona, G., Pinto, L., Pagliari, D., Passoni, D., Gini, R., 2014. Experimental analysis of different software packages for orientation and digital surface modelling from UAV images. Earth Sci. Inform. 7, 97-107.

Tilly, N., Hoffmeister, D., Cao, Q., Huang, S., LenzWiedemann, V., Miao, Y. \& Bareth, G., 2014. Multitemporal crop surface models: accurate plant height measurement and biomass estimation with terrestrial laser scanning in paddy rice. Journal of Applied Remote Sensing 8 (1), 83671-83693.

Verhoeven, G., 2011. Taking ComputerVision Aloft Archaeological Three-dimensional Reconstructions from Aerial Photographswith PhotoScan Archaeological Prospection 18 (1), 67-73. 